\title{
Atraumatic Rupture of the Distal Median Nerve
}

\author{
Liam Bibo $^{1 *}$, Alex 0'Beirne ${ }^{\mathbf{1}}$ and Matthew Lawson-Smith ${ }^{1,2}$ \\ ${ }^{1}$ Department of Hand Surgery, Fremantle Hospital, Fremantle, Australia \\ ${ }^{2}$ University of Notre Dame, Fremantle, Australia \\ *Corresponding Author: Liam Bibo, Department of Hand Surgery, Fremantle \\ Hospital, Fremantle, Australia.
}

Received: December 21, 2020

Published: January 16, 2020

(C) All rights are reserved by Liam Bibo.,

et al.

\begin{abstract}
Carpal tunnel syndrome (CTS) is the most common compressive neuropathy affecting the upper extremity. We present a rare case of atraumatic rupture of the median nerve in a patient with electrophysiology proven severe left distal median nerve neuropathy secondary to CTS.
\end{abstract}

Keywords: Median Nerve; Carpal Tunnel Syndrome; Hand; Surgery

\section{Abbreviations}

CTS: Carpal Tunnel Syndrome; CT: Computed Tomography.

\section{Introduction}

Carpal tunnel syndrome (CTS) is the most common compressive neuropathy affecting the upper extremity, present in approximately $3 \%$ to $6 \%$ of adults [1]. The key elements include paraesthesia of the hand in the median nerve distribution, nocturnal wakening, thenar atrophy, a positive Phalen test, loss of two-point discrimination and a positive Tinel's sign [1]. Whilst spontaneous atraumatic tendon rupture is well documented in the literature, we are unaware of a nerve particularly a median nerve rupture being reported [2]. We present a rare case of atraumatic rupture of the median nerve in a patient with electrophysiology proven severe left distal median nerve neuropathy, secondary to CTS.

\section{Case Report}

A 55 year old woman presented with a two year history of pins and needles in her left hand and pain which wakes her at night, which had developed approximately four months post suffering a distal radius fracture on the same side from a fall on outstretched hand. Her fracture was treated non-operatively with casting, without complication. Her medical history is significant for a 10 packyear history of smoking but was otherwise in good health. She works as an occupational health nurse.

On examination she had adductor pollicis brevis wasting and weakness and was Durkan's compression test positive. Nerve conduction studies confirmed the clinical diagnosis of CTS and she was subsequently booked for surgical decompression. She had not had previous corticosteroid injection of the carpal tunnel. On the day of her procedure the patient presented with quite marked swelling around the volar surface of her wrist. She denied any recent trauma to her wrist. She did report an acute episode of left wrist pain whist lawn mowing on the day prior to her operation, however, her hand symptoms were unchanged.

Her surgery proceeded with open carpal tunnel decompression technique, performed under sedation with tourniquet control and using loupes. Local anaesthetic was infiltrated just superficial to the skin at the site of surgery. After the transverse carpal ligament 
was released a large volume of synovial fluid was released and the nerve exposed. On inspection of the median nerve it was found to be transected, with no signs of iatrogenic injury (Figure 1). The patient's care was then transferred to a hand surgeon with a specialist interest in peripheral nerve surgery, with her procedure performed the same day.

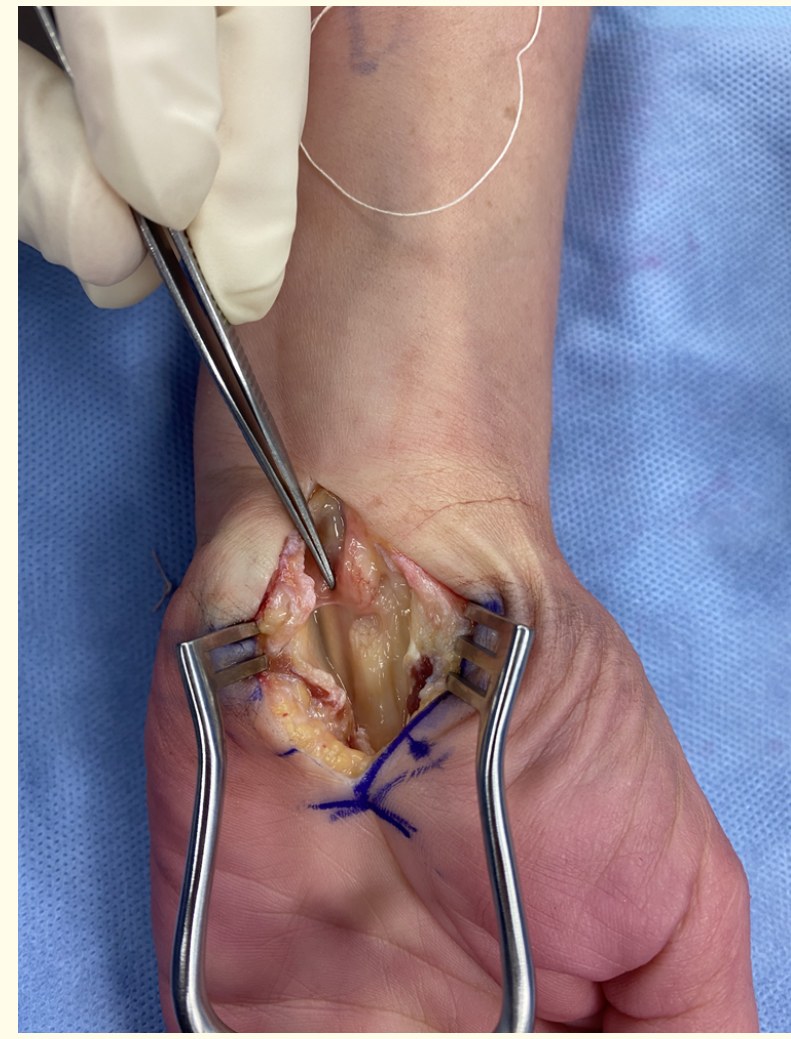

Figure 1: PExposure of the transected median nerve.

The wound was extended proximally and distally, with significant florid tenosynovitis involving all flexor tendons and a rupture of the median nerve in zone $\mathrm{V}$ was identified. The median nerve had an uneven ragged rupture with disruption of the fascicles at different levels. The nerve was debrided to healthy fascicles, with a $5 \mathrm{~mm}$ gap present. The surgeon proceeded with a primary repair of the median nerve using 8/0 nylon (Figure 2).

Histopathology identified acute synovitis of the flexor synovium and nerve with acute inflammation. The microbiology and microscopy results were unremarkable.

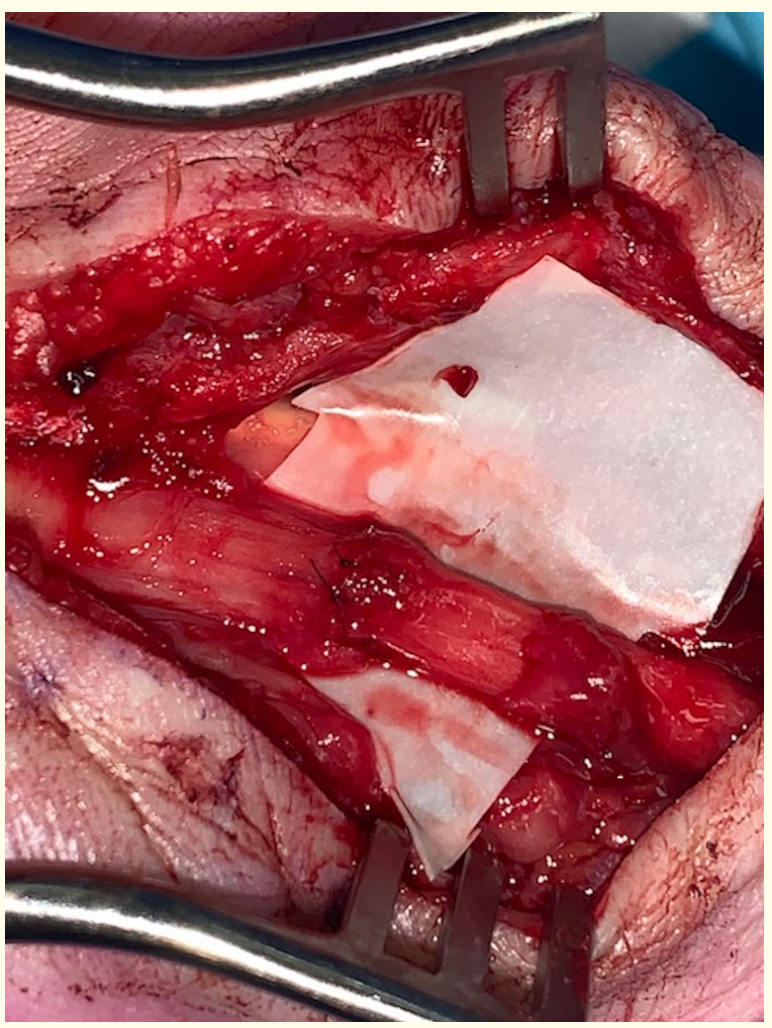

Figure 2: Primary repair of the median nerve.

On post-operative review the patient had altered sensation in the thumb, index and middle fingers, and normal sensation in her ring finger on Semmes-Weinstein testing. She has good movement in all of her fingers and thumb. A post-operative computed tomography (CT) scan did not identify a volar abnormality of the distal radius or any bone fragments within the carpal tunnel (Figure 3).

\section{Discussion}

A review of the literature did not identify any documented cases of atraumatic rupture of the median nerve. Only three cases of atraumatic nerve rupture of the upper limb were found, all were of the ulnar nerve and associated with rheumatoid arthritis [3]. The patient did not report any symptoms suggestive of rheumatoid arthritis. Histopathology did identify acute synovitis of the flexor synovium.

CTS has been well described as one of the major complications of distal radius fracture, and its incidence has been reported between $3.3 \%$ and $17.2 \%$ [4]. The patient had suffered a minimally 


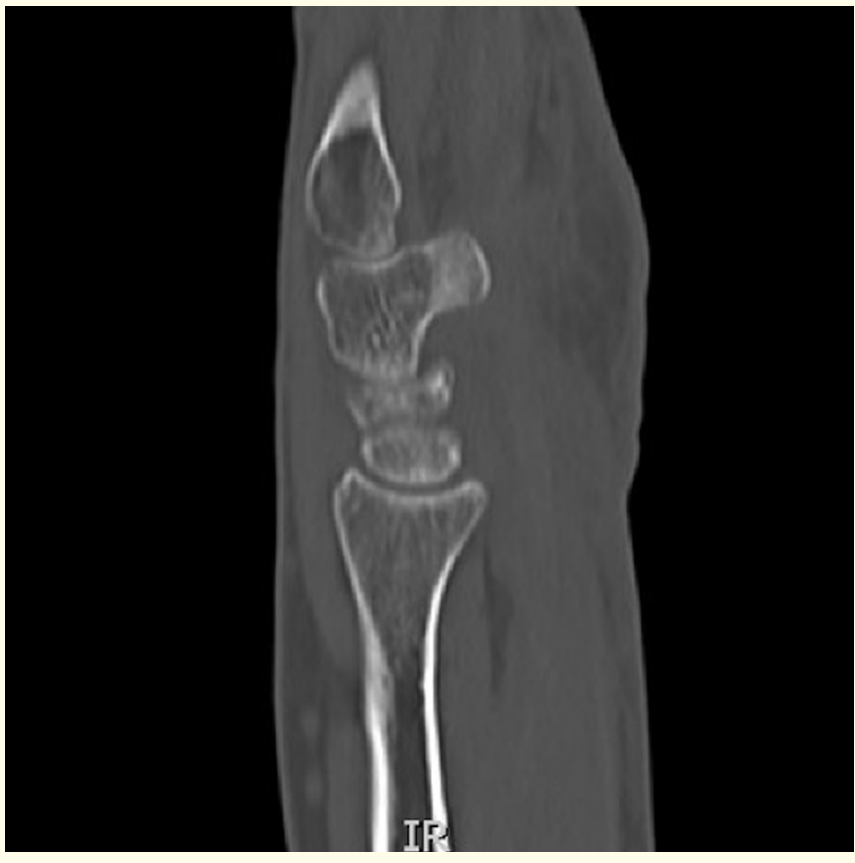

Figure 3: CT scan of wrist, identifying no volar bony abnormality.

impacted and dorsally displaced transverse fracture of the distal radius prior to the onset of her hand symptoms, with her fracture managed non-operatively. In patients with CTS, when the intracarpal canal interstitial pressure rises above a critical threshold pressure, capillary blood flow is reduced below the level required for median nerve viability [5]. Ischaemic injury of the nerve may account for subsequent median nerve rupture in this case.

The acute episode of wrist pain prior to her procedure and the abundance of synovial fluid at the time of operation was likely consistent with atraumatic nerve rupture. The surgical exposure of the median nerve was uncomplicated and routine precautions were taken to protect the nerve when releasing the transverse carpal ligament under tourniquet control. The rupture pattern of the median nerve with disruption of the fascicles at different levels supported that the rupture was not iatrogenic in nature.

\section{Conclusion}

The authors suggest the most likely cause for median nerve rupture was secondary to nerve inflammation from synovitis, nerve ischaemia subsequent to compressive neuropathy or nerve injury from a displaced spicule of bone. This case demonstrates a very rare form of median nerve injury, to our knowledge not previously documented in published literature.

\section{Conflict of Interest}

Author declarations of interests: none.

This research did not receive any specific grant from funding agencies in the public, commercial, or not-for-profit sectors.

Informed consent was gained from the individual patient involved, to submit this case study for publication.

\section{Bibliography}

1. Calandruccio JH and Thompson NB. "Carpal Tunnel Syndrome: Making Evidence-Based Treatment Decisions”. Orthopedic Clinics of North America 49.2 (2018): 223-229.

2. Rada EM., et al. "Spontaneous atraumatic extensor pollicis longus rupture in the nonrheumatoid population". Eplasty 13 (2013): e11.

3. Kaneko A., et al. "Attrition rupture of ulnar nerve in a patient with rheumatoid elbow arthritis: A case report". Medicine (Baltimore) 97.17 (2018): e0535.

4. Itsubo T., et al. "Differential onset patterns and causes of carpal tunnel syndrome after distal radius fracture: a retrospective study of 105 wrists". Journal of Orthopaedic Science 15.4 (2010): 518-523.

5. Thurston A. "Carpal tunnel syndrome". Orthopaedic Trauma 27.5 (2013): 332-341.

\section{Assets from publication with us}

- Prompt Acknowledgement after receiving the article

- Thorough Double blinded peer review

- Rapid Publication

- Issue of Publication Certificate

- High visibility of your Published work

Website: https://www.actascientific.com/

Submit Article: https://www.actascientific.com/submission.php Email us: editor@actascientific.com

Contact us: +919182824667 